\title{
Model Reduction for RF MEMS Simulation
}

\author{
David S. Bindel ${ }^{1}$, Zhaojun Bai ${ }^{2}$, and James W. Demmel ${ }^{3}$ \\ ${ }^{1}$ Department of Electrical Engineering and Computer Science \\ University of California at Berkeley \\ Berkeley, CA 94704 \\ dbindel@eecs.berkeley.edu \\ 2 Department of Computer Science \\ University of California at Davis \\ Davis, CA 95616 \\ bai@cs.ucdavis.edu \\ 3 Department of Electrical Engineering and Computer Science and \\ Department of Mathematics \\ University of California at Berkeley \\ Berkeley, CA 94704 \\ demmel@eecs.berkeley.edu
}

\begin{abstract}
Radio-frequency (RF) MEMS resonators, integrated into CMOS chips, are of great interest to engineers planning the next generation of communication systems. Fast simulations are necessary in order to gain insights into the behavior of these devices. In this paper, we discuss two structure-preserving model-reduction techniques and apply them to the frequency-domain analysis of two proposed MEMS resonator designs.
\end{abstract}

\section{Introduction}

Radio-frequency (RF) electromechanical resonators used in frequency references and filters are critical to modern communication systems. For RF engineers using quartz, ceramic, and surface acoustic wave devices, surface-micromachined MEMS resonators in development offer an attractive alternative. Because they can be integrated into CMOS, MEMS resonators have the potential to cost less area, power, and money than existing alternatives [10]. Such integrated high-frequency filters could qualitatively change the design of cell phones, making it possible to build a cheap phone to work with multiple networks operating at different carrier frequencies, and lowering power and size constraints to the point that inexpensive "Dick Tracy" watch phones could be a reality.

Researchers working on integrated MEMS resonators currently rely on a trial-anderror design process. There is a clear need for quick, accurate simulations to minimize the labor and fabrication costs of trial-and-error design. Lumped-parameter models and simple modal analyses do not adequately describe subtle continuum-level effects and mode interactions which strongly affect device behavior. We therefore rely on modelreduction methods to perform frequency-response computations quickly.

Energy losses in high-frequency resonators are critical to designers. The quality factor $(Q)$, a ratio of energy stored in a resonator to energy lost in each cycle, must be high for a resonator to be useful in a filter or frequency reference. Despite their 
importance, the details of energy loss in MEMS resonators are not well understood, and accurate loss modeling remains an area of active research. Among other mechanisms, energy can be lost through viscous damping, through thermoelastic effects, or through elastic waves carrying energy away into the substrate [8]. Each mechanism leads to a different structure in the model equations. A mechanical system subject to viscous damping is modeled by a second-order system of equations with a nonzero first-order term; the thermoelastic equations are coupled first- and second-order equations; while infinite-domain problems can be modeled by a coordinate transformation resulting in complex symmetric mass and stiffness matrices. Here we consider viscous and anchor losses. Viscous loss models are relatively common, but to our knowledge, ours is the first physical model of anchor loss in a bulk-mode MEMS resonator.

In this paper, we describe two model reduction techniques for computing the frequency response of MEMS resonators. We first describe how to reduce second order systems using a Second-Order Arnoldi (SOAR) algorithm which preserves the secondorder structure of the equations; we use this technique to simulate a checkerboard-shaped mechanical filter. We then describe how we model infinite-domain problems using a perfectly-matched layer (PML), and describe a model-reduction technique which preserves the structure of the PML; we use this technique to study the frequency response of a disk resonator.

\section{Model Reduction for Second-Order Systems}

\subsection{Second-Order Systems and SOAR}

Since they are partly mechanical, most MEMS models are naturally second-order in time. Depending on the device, there may also be equations which are first order in time or algebraic, such as the equations for heat transport or for electrostatics. The linearized equations for the coupled system can be written as a continuous time-invariant singleinput single-output second-order system

$$
\Sigma_{N}:\left\{\begin{aligned}
M \ddot{q}(t)+D \dot{q}(t)+K q(t) & =b u(t) \\
y(t) & =l^{T} q(t)
\end{aligned}\right.
$$

with initial conditions $q(0)=q_{0}$ and $\dot{q}(0)=\dot{q}_{0}$. Here $t$ is time; $q(t) \in \mathcal{R}^{N}$ is a vector of state variables; $N$ is the state-space dimension; $u(t) \in \mathcal{R}$ and $y(t) \in \mathcal{R}$ are the input force and output measurement functions; $M, D, K \in \mathcal{R}^{N \times N}$ are system mass, damping, and stiffness matrices; and $b$ and $l$ are input distribution and output measurement vectors. The state-space dimension $N$ of the system $\Sigma_{N}$ is typically large and it can be slow to use for practical analysis and design. Therefore, model-order reduction techniques are needed to construct compact models which both retain important properties of the original model and are fast to simulate.

A common approach to model-order reduction for the second-order model $\Sigma_{N}$ is to add variables for the velocities $\dot{q}(t)$, and so create a first-order model of twice the dimension of the original. Then the first-order model is reduced. However, the reduced first-order model may not correspond to any second-order model, and may lack properties 
of the original model, such as stability and passivity. There have been a number of efforts toward such structure-preserving model-order reduction methods; we focus on a subspace projection method based on a second-order Arnoldi (SOAR) procedure that not only preserves the second-order structure, but also matches the same number of moments as the standard Arnoldi-based Krylov subspace method via linearization for about the same amount of work. For the rest of this subsection, we present a SOAR-based model-order reduction method from the view discussed in [2].

The second-order system $\Sigma_{N}$ is represented in the time domain in 2.1). Equivalently, one can represent the system in the frequency domain via the Laplace transform. Assuming homogeneous initial conditions, the frequency-domain input $U(s)$ and output $Y(s)$ are related by the transfer function

$$
H(s)=l^{T}\left(s^{2} M+s D+K\right)^{-1} b=\sum_{\ell=0}^{\infty} m_{\ell}\left(s-s_{0}\right)^{\ell},
$$

where the coefficients $m_{\ell}$ in the Taylor series about $s_{0}$ are called moments. The moments can be written as $m_{\ell}=l^{T} r_{\ell}$, where the second-order Krylov vector sequence $\left\{r_{\ell}\right\}$ is defined by the following recurrence:

$$
\begin{aligned}
r_{0} & =\widehat{K}^{-1} b \\
r_{1} & =-\widehat{K}^{-1} \widehat{D} r_{0} \\
r_{\ell} & =-\widehat{K}^{-1}\left(\widehat{D} r_{\ell-1}+M r_{\ell-2}\right) \quad \text { for } \quad \ell=2,3, \ldots
\end{aligned}
$$

where $\widehat{K}=s_{0}^{2} M+s_{0} D+K$ and $\widehat{D}=2 s_{0} M+D$.

The subspace $\mathcal{G}_{n}=\operatorname{span}\left\{r_{\ell}\right\}_{\ell=0}^{n-1}$ is called a second-order Krylov subspace. Let $Q_{n}$ be an orthonormal basis of $\mathcal{G}_{n}$. We seek an approximation $q(t) \approx Q_{n} z(t) \in \mathcal{G}_{n}$; this is often referred to as a change of state coordinates. By imposing the Galerkin condition:

$$
M Q_{n} \ddot{z}(t)+D Q_{n} \dot{z}(t)+K Q_{n} z(t)-b u(t) \perp \mathcal{G}_{n},
$$

we obtain the following structure-preserving reduced-order model:

$$
\Sigma_{n}:\left\{\begin{array}{rl}
M_{n} \ddot{z}_{n}(t)+D_{n} \dot{z}_{n}(t)+K_{n} z(t) & =b_{n} u(t) \\
\tilde{y}(t) & =l_{n}^{T} z(t)
\end{array},\right.
$$

where $M_{n}=Q_{n}^{T} M Q_{n}, D_{n}=Q_{n}^{T} D Q_{n}, K_{n}=Q_{n}^{T} K Q_{n}, b_{n}=Q_{n}^{T} b$ and $l_{n}=Q_{n}^{T} l$. It can be shown that the first $n$ moments of the reduced model $\Sigma_{n}$ agree with those of the original model $\Sigma_{N}$. Furthermore, if $M, D$, and $K$ are symmetric and $b=l$, then the first $2 n$ moments of the models are the same. This method has the same order of approximation as the standard Arnoldi-based Krylov subspace method via linearization.

We produce an orthonormal basis $Q_{n}$ for the second-order Krylov subspace $\mathcal{G}_{n}$ using a Second-Order ARnoldi (SOAR) procedure proposed by Su and Craig [12] and further improved by Bai and Su [3]. At step $j$, the algorithm computes

$$
r=-\widehat{K}^{-1}\left(\widehat{D} q_{j}+M w\right)
$$

where $w$ is an auxiliary vector computed at the end of the previous step. Then $r$ is orthogonalized against $Q_{j}$ and normalized to produce $q_{j+1}$ and $w$ is recomputed. 
In contrast to the standard Arnoldi algorithm, the transformed matrix triplet $(M, \widehat{D}, \widehat{K})$ is used to generate an orthonormal basis $Q_{n}$ of $\mathcal{G}_{n}$, but the original matrix triplet $(M, D, K)$ is directly projected onto the subspace $\mathcal{G}_{n}$ to define the reduced-order model $\Sigma_{n}$. By explicitly formulating the matrices $M_{n}, D_{n}$ and $K_{n}$, essential structures of $M, D$ and $K$ are preserved. For example, if $M$ is symmetric positive definite, so is $M_{n}$. As a result, we can preserve the stability, symmetry and physical meaning of the original second-order model $\Sigma_{N}$. This is in the same spirit as the widely-used PRIMA algorithm for passive reduced-order modeling of linear dynamical systems arising from interconnect analysis in circuit simulations [11].

The SOAR-based model-order reduction algorithm has many desirable properties compared to the linearization approach. The reduced system $\Sigma_{n}$ not only preserves the second-order structure, but also matches the same number of moments as the standard method of projecting a linearized system onto a basis of $n$ Arnoldi vectors. SOAR-based algorithms require less space and fewer flops (for a subspace of the same dimension), and also provide more accurate results.

\subsection{Modeling of a Checkerboard Resonator}

As an application, we build a reduced-order model from a finite element simulation of a prototype MEMS filter. The goal for this device is to produce a high-frequency bandpass filter to replace, for example, the surface acoustic wave (SAW) devices used in cell phones. The device (Figure 1) consists of a checkerboard of silicon squares which are linked at the corners [6]. The "checkers" are held at a fixed voltage bias relative to the drive and sense electrodes placed around the perimeter. A radio-frequency (RF) voltage variation on drive electrodes at the northwest corner creates an electrostatic force which causes the device to move in plane. The motion induces changes in capacitance at the sense electrodes at the southwest corner of the device. The induced motion is typically very small; the checker squares are two microns thick and tens of microns on a side, and the maximum displacement is on the order of tens of nanometers.
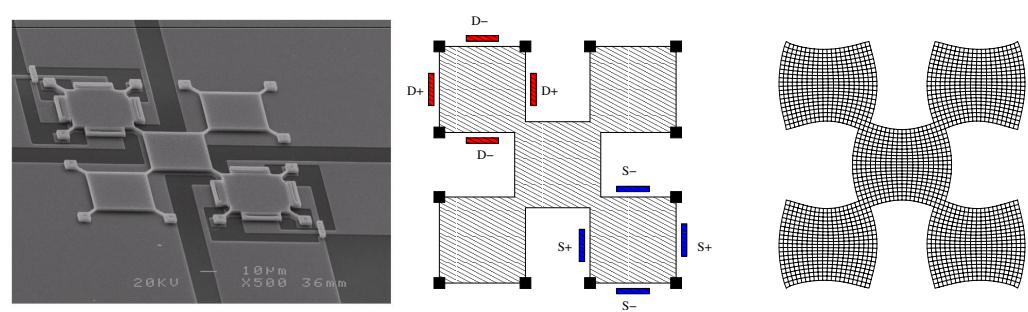

Fig. 1. Illustration of a checkerboard resonator. The SEM picture (left) shows a fabricated device, and the simulation (right) shows one resonant mode excited during operation. The motion is excited at the northwest corner and sensed at the southeast corner (center)

A single square pinned at the corners exhibits a "Lamé mode." If the interaction between squares was negligible, the system would have a five-fold eigenvalue corresponding to independent Lamé-mode motions for each square. The coupling between 

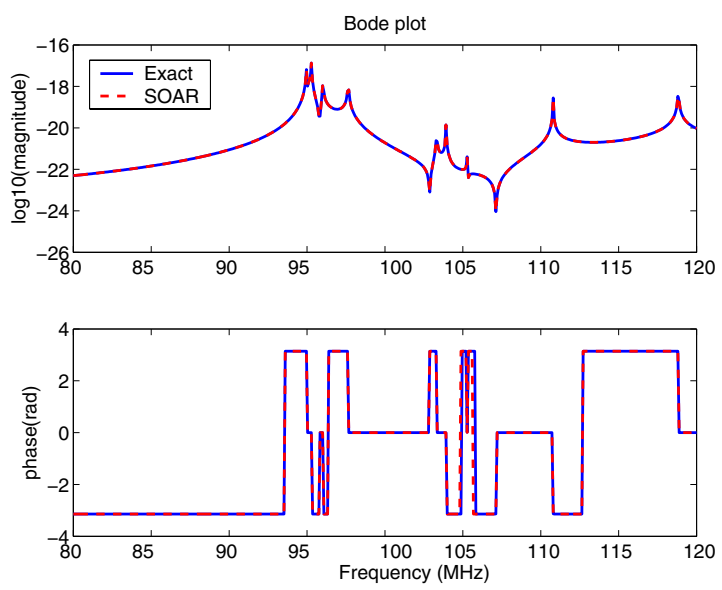

Fig. 2. Bode plots from finite element model and reduced order model for a 3-by-3 checkerboard resonator

the squares causes the five eigenvalues to split, so there are several poles near each other; consequently, the array has low motional resistance near the target frequency. The same idea of using weakly coupled arrays has also been applied to other RF resonator designs [9].

We model the checkerboard with linear 2D plane stress elements and an empirical viscous damping model. Even for a small mesh $(N=3231)$, model reduction is beneficial. Using a reduced model with 150 degrees of freedom, we obtain a Bode plot which is visually indistinguishable from the plot for the unreduced system (Figure 2). We have also created a visualization tool which designers can use to see the forced motion at different frequencies (Figure 3). With a reduced model, we can compute the shape of the motion at a specified frequency within tens of milliseconds instead of seconds, quickly enough for the user to interactively scan through frequencies of interest. Designers can use these visualizations to build intuition about different strategies for constructing anchors, connecting resonator components, and placing drive and sense electrodes.

\section{Model Reduction for Perfectly-Matched Layers}

\subsection{Perfectly-Matched Layers and Symmetry-Preserving Projection}

In several high $\mathrm{MHz}$ or $\mathrm{GHz}$ frequency resonator designs, the dominant loss mechanism appears to be radiation of elastic energy through anchors. In these designs, the resonating device is so much smaller than the silicon substrate that waves leaving the anchor are so attenuated by material losses as to render negligible any reflections from the sides of the chip. That is, the bulk of the chip can be modeled as a semi-infinite medium. We model this semi-infinite domain using a perfectly matched layer (PML), which absorbs waves at any angle of incidence [4]. 


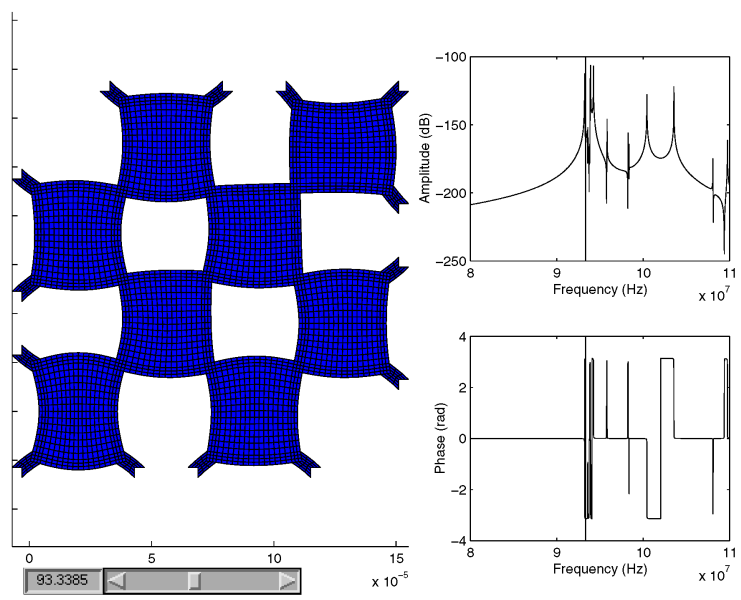

Fig. 3. Screenshot of a visualization tool for observing forced response shapes for in-plane resonators. Using a reduced model, the tool can compute and plot the response shape quickly enough for interactive use

Bérenger invented the perfectly matched layer for problems in electromagnetics [5], but it was almost immediately re-interpreted as a complex-valued change of coordinates which can be applied to any linear wave equation [14|13]. The weak form of the PML equations for time-harmonic linear elasticity [4] is

$$
\int_{\Omega} \tilde{\epsilon}(w)^{T} D \tilde{\epsilon}(u) \tilde{J} d \Omega-\omega^{2} \int_{\Omega} \rho w \cdot u \tilde{J} d \Omega=\int_{\Gamma} w \cdot t d \Gamma
$$

where $u$ and $w$ are displacement and weight functions on the domain $\Omega ; t$ is a traction defined on the boundary $\Gamma ; \rho$ and $D$ are the mass density and the elasticity matrix; $\tilde{\epsilon}$ is a transformed strain vector; and $\tilde{J}$ is the Jacobian determinant of the PML transformation. This weak form is nearly identical to the standard weak form of linear elasticity, and when we discretize with finite elements, we have the familiar system

$$
\left(K-\omega^{2} M\right) q=b
$$

where $K$ and $M$ are now complex symmetric.

To make the attenuation through the PML frequency-independent, the coordinate transformations in [4] are frequency-dependent. Using a frequency-dependent transformation, the transfer function is given by

$$
H(i \omega)=l^{T}\left(K(\omega)-\omega^{2} M(\omega)\right)^{-1} b
$$

and complex resonances satisfy the nonlinear eigenvalue equation

$$
\operatorname{det}\left(K(\omega)-\omega^{2} M(\omega)\right)=0 .
$$

However, when the frequency range of interest is not too wide - when the maximum and minimum frequency considered are within a factor of two or three of each other - 
the parameters of the PML may be chosen once to give acceptable attenuation over the desired frequency range. So for $\omega$ near enough to $\omega_{0}$, we approximate $H(i \omega)$ by

$$
H_{0}(i \omega)=l^{T}\left(K\left(\omega_{0}\right)-\omega^{2} M\left(\omega_{0}\right)\right)^{-1} b
$$

Similarly, the nonlinear eigenvalue problem (3.9) may be replaced by a (generalized) linear eigenvalue problem.

In [1], Arbenz and Hochstenbach suggest a variant of the Jacobi-Davidson algorithm for complex symmetric eigenvalue problems. In this method, the standard Rayleigh quotient estimate of the eigenvalue, $\theta(v)=\left(v^{H} K v\right) /\left(v^{H} M v\right)$, is replaced by $\psi(v)=\left(v^{T} K v\right) /\left(v^{T} M v\right)$. Assuming $v^{T} M v \neq 0, \psi(v)$ converges quadratically to an eigenvalue of a complex-symmetric pencil as $v$ approaches an eigenvector, while $\theta(v)$ converges only linearly. The extra order of accuracy comes from the symmetry of the quadratic forms

$$
u^{T} M v=v^{T} M u \text { and } u^{T} K v=v^{T} K u .
$$

We wish to maintain this symmetry in our reduced-order models as well.

To build a reduced-order model, we generate a basis $V$ for the Krylov subspace $\mathcal{K}_{n}\left(\left(K\left(\omega_{0}\right)-\omega_{0}^{2} M\left(\omega_{0}\right)\right)^{-1}, b\right)$ with the standard Arnoldi algorithm. We then construct a symmetry-preserving reduced-order model by choosing an orthonormal projection basis $W$ such that

$$
\operatorname{span}(W)=\operatorname{span}([\operatorname{Re}(V), \operatorname{Im}(V)]) .
$$

The reduced model will be at least as accurate as the usual Arnoldi projection, but will maintain the complex symmetry of the original system. This reduced model also corresponds to a Bubnov-Galerkin discretization of the PML equation with a set of real-valued global shape functions.

\subsection{Modeling of a Disk Resonator}

As an example of PML model reduction, we study the anchor loss in a disk-shaped MEMS resonator presented in [15] (Figure 4). The disk sits on a silicon post, and differences in voltage between the disk and the surrounding drive electrodes pull the disk rim outward, exciting an axisymmetric motion. The disk is driven near the frequency of

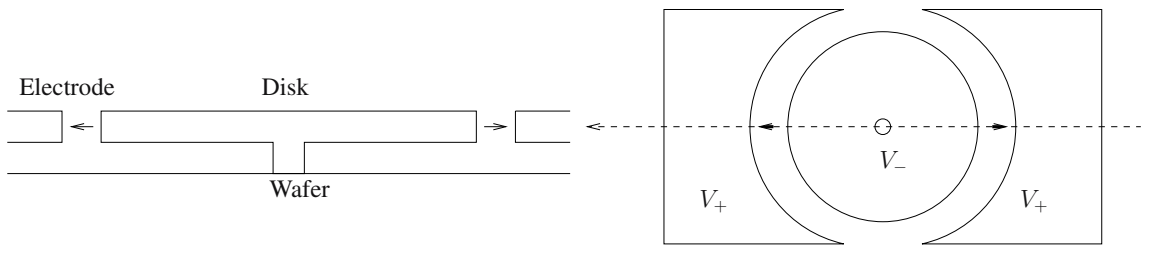

Fig. 4. Schematic of the Michigan disk resonator. An overhead view (right) shows the arrangement of the resonating disk and the electrodes which force it. An idealized cross-section (left) is used in an axisymmetric simulation, where the wafer substrate is treated as semi-infinite using a perfectly matched layer 
the first or second radial mode, and the motion is sensed capacitively by electrodes on the surface of the substrate.

We model this resonator using axisymmetric finite elements, with a PML region to mimic the effects of a semi-infinite substrate. We wish to use this model to study sharpness of resonant peaks, as quantified by the quality factor $Q$. For a resonant frequency $\omega=\alpha+i \beta, Q$ is given by $\alpha /(2 \beta)$. For typical RF applications, $Q$ values of at least 1000 are required; higher values are better.

Because the discretization errors in the real and imaginary parts of the computed eigenvalues are about the same size, we must resolve $\omega$ to an overall relative error significantly less than $Q^{-1}$ before the computed $Q$ converges. Consequently, we use a relatively fine mesh of high-order (bicubic) elements with 57475 unknowns in order to resolve the behavior of this device. With this mesh, the computed $Q$ value of the second radial mode was 6250; the measured value of an actual device was 7330 (in vacuum).

Because we expect a single sharp peak to dominate the response in the frequency range of interest, we might expect a single step of shift-invert Arnoldi with a good shift to produce a reasonable approximation to $H_{0}(i \omega)$. If the device possesses two nearly coincident modes, then two Arnoldi iterations are needed. When the two modes are very close, they strongly interact, and any reduced model must account for both. Furthermore, when multiple poles are involved, the $Q$ values provided by eigenvalue computations no longer provide a complete picture of the behavior near a resonant peak. To understand the response, we use a reduced model with three Arnoldi vectors (two steps of Arnoldi plus a starting vector), which approximates the full model very closely near the peak (Figure 5). Though the peak for one of the modes has negligible magnitude compared to the peak for the other mode, the interaction of the two modes strongly affects the sharpness of the dominant mode peak: for values of the disk thickness where the two modes most closely coincide, the computed $Q$ value for the dominant-mode peak varies over five orders of magnitude [7].

\section{Conclusion}

In this paper, we have described two model reduction techniques: a method based on the SOAR (Second-Order ARnoldi) algorithm, which preserves the second-order structure of a full system; and a method which uses a standard Arnoldi basis in a novel way to preserve the complex-symmetric structure of an infinite-domain problem approximated by a perfectly matched layer. We have illustrated the utility of these methods by computing the frequency-response behavior of two real designs of very high-frequency MEMS resonators. In each case, the interactions between multiple resonant modes proves critical, so that naive projection onto a single mode is inadequate for exploring even the local frequency-response behavior.

As we continue to study energy loss mechanisms in RF MEMS, we expect model reduction to play an even more critical role. For example, to study anchor loss in the checkerboard resonator, we plan to build a 3D finite element model with perfectly matched layers below the anchors; this model will be much larger than the 2D model described in this paper. We are also interested in structure-preserving model reduction for thermoelastic damping, which is an important loss mechanism in at least some 

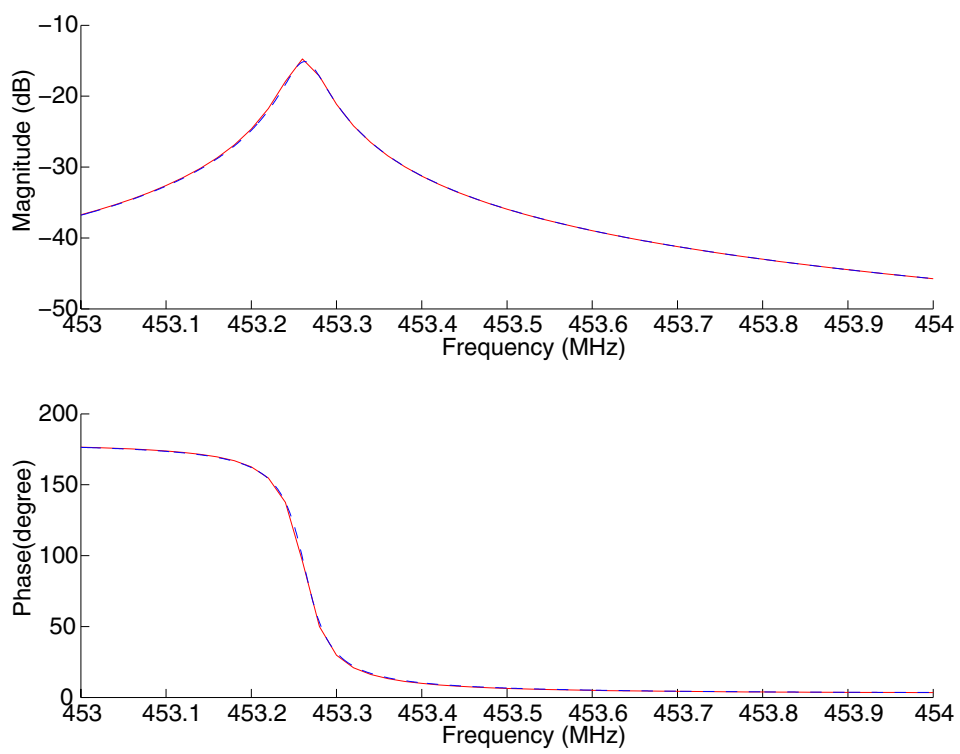

Fig. 5. Bode plot for a disk resonator with nearly coincident modes. The exact model (solid) matches the reduced model (dashed) produced from two Arnoldi steps

types of flexural resonators [8]. Finally, to more quickly study how variations in device geometry affect performance, we plan to investigate methods for building parameterized reduced-order models and reusable reduced-order models for substructures.

\section{Acknowledgements}

Sunil Bhave and Roger Howe of the Berkeley EECS department proposed and fabricated the checkerboard resonator design, and have provided valuable feedback during the development of our simulations. The study of resonator loss mechanisms is collaborative work with Sanjay Govindjee and Tsuyoshi Koyama of the Berkeley Civil Engineering department.

\section{References}

1. P. Arbenz and M. E. Hochstenbach. A Jacobi-Davidson method for solving complex symmetric eigenvalue problems. SIAM J. Sci. Comp., 25(5):1655-1673, 2004.

2. Z. Bai and Y. Su. Dimension reduction of second-order dynamical systems via a second-order Arnoldi method. SIAM J. Sci. Comp., 2004. to appear.

3. Z. Bai and Y. Su. SOAR: A second-order Arnoldi method for the solution of the quadratic eigenvalue problem. SIAM J. Matrix Anal. Appl., 2004. to appear.

4. U. Basu and A. Chopra. Perfectly matched layers for time-harmonic elastodynamics of unbounded domains: theory and finite-element implementation. Computer Methods in Applied Mechanics and Engineering, 192:1337-1375, 2003. 
5. J.-P. Bérenger. A perfectly matched layer for the absorption of electromagnetic waves. Journal of Computational Physics, 114(2):185-200, 1994.

6. S. Bhave, D. Gao, R. Maboudian, and R. T. Howe. Fully differential poly-SiC Lamé mode resonator and checkerboard filter. In Proceedings of MEMS 05, Miami, FL, January 2005.

7. D. S. Bindel, E. Quévy, T. Koyama, S. Govindjee, J. W. Demmel, and R. T. Howe. Anchor loss simulation in resonators. In Proceedings of MEMS 05, Miami, FL, January 2005.

8. R. N. Candler, H. Li, M. Lutz, W.-T. Park, A. Partridge, G. Yama, and T. W. Kenny. Investigation of energy loss mechanisms in micromechanical resonators. In Proceedings of Transducers 03, pages 332-335, Boston, June 2003.

9. M. U. Demirci, M. A. Abdelmoneum, and C. T.-C. Nguyen. Mechanically corner-coupled square microresonator array for reduced series motional resistance. In Proc. of the 12th Intern. Conf. on Solid State Sensors, Actuators, and Microsystems, pages 955-958, Boston, June 2003.

10. C. T.-C. Nguyen. Vibrating RF MEMS for low power wireless communications. In Proceedings of the 2001 International MEMS Workshop (iMEMS01), pages 21-34, Singapore, July 2001.

11. A. Odabasioglu, M. Celik, and L. T. Pileggi. PRIMA: passive reduced-order interconnect macromodeling algorithm. IEEE Trans. Computer-Aided Design of Integrated Circuits and Systems, 17:645-654, 1998.

12. T.-J. Su and R. R. Craig Jr. Model reduction and control of flexible structures using Krylov vectors. J. of Guidance, Control and Dynamics, 14:260-267, 1991.

13. F. Teixeira and W. Chew. Complex space approach to perfectly matched layers: a review and some new developments. International Journal of Numerical Modelling, 13(5):441-455, 2000.

14. E. Turkel and A. Yefet. Absorbing PML boundary layers for wave-like equations. Applied Numerical Mathematics, 27(4):533-557, 1998.

15. J. Wang, Z. Ren, and C. T-C. Nguyen. Self-aligned $1.14 \mathrm{GHz}$ vibrating radial-mode disk resonators. In Proceedings of Transducers 03, pages 947-950, 2003. 